
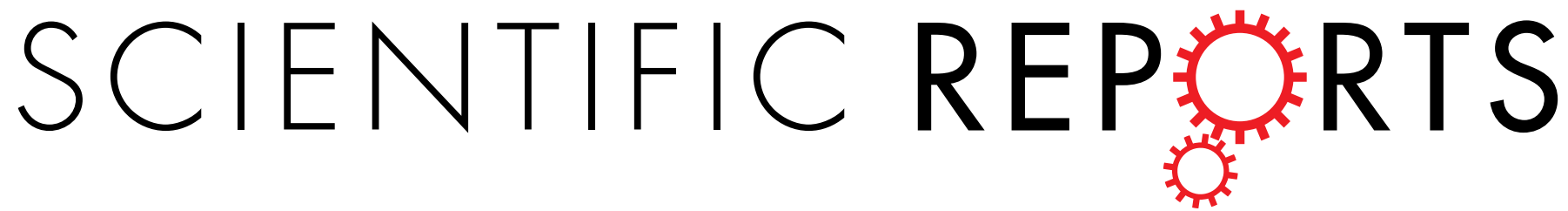

\title{
OPEN Statins do not increase Markers of Cerebral Angiopathies in patients with Cardioembolic Stroke
}

Received: 2 October 2017

Accepted: 11 January 2018

Published online: 24 January 2018
Joan Martí-Fàbregas ${ }^{1}{ }^{1}$, Santiago Medrano-Martorell², Elisa Merino ${ }^{3}$, Luis Prats-Sánchez ${ }^{1}$, Rebeca Marín ${ }^{1}$, Raquel Delgado-Mederos ${ }^{1}$, Pol Camps-Renom ${ }^{1}$, Alejandro MartínezDomeño ${ }^{1}$, Manuel Gómez-Choco ${ }^{5}$, Lidia Lara ${ }^{6}$, Ignacio Casado-Naranjo7 , David Cánovas ${ }^{8}$, Maria José Torres ${ }^{9}$, Marimar Freijo ${ }^{10}$, Ana Calleja ${ }^{11}$, Yolanda Bravo ${ }^{12}$, Dolores Cocho ${ }^{13}$, Ana Rodríguez-Campello ${ }^{14}$, Beatriz Zandio ${ }^{15}$, Blanca Fuentes ${ }^{16}$, Alicia de Felipe ${ }^{17}$, Laura Llull ${ }^{18}$, José Maestre ${ }^{19}$, María Hernández ${ }^{20}$, Moisès Garcés ${ }^{21}$, Ana Maria De Arce-Borda ${ }^{22}$, Ernest Palomeras $^{23}$, Manuel Rodríguez-Yáñez ${ }^{24}$, Inma Díaz-Maroto ${ }^{25}$, Marta Serrano ${ }^{26}$, Jéssica Fernández-Domínguez ${ }^{27}$, Jordi Sanahuja ${ }^{28}$, Francisco Purroy ${ }^{28}$, Marialuisa Zedde ${ }^{29}$, Jordi Delgado-Mengual ${ }^{30}$ \& Ignasi Gich ${ }^{4}$

We investigated whether pre-treatment with statins is associated with surrogate markers of amyloid and hypertensive angiopathies in patients who need to start long-term oral anticoagulation therapy. A prospective multicenter study of patients naive for oral anticoagulants, who had an acute cardioembolic stroke. MRI was performed at admission to evaluate microbleeds, leukoaraiosis and superficial siderosis. We collected data on the specific statin compound, the dose and the statin intensity. We performed bivariate analyses and a logistic regression to investigate variables associated with microbleeds. We studied 470 patients (age $77.5 \pm 6.4$ years, $43.7 \%$ were men), and $193(41.1 \%)$ of them received prior treatment with a statin. Microbleeds were detected in 140 (29.8\%), leukoaraiosis in $388(82.5 \%)$ and superficial siderosis in 20 (4.3\%) patients. The presence of microbleeds, leukoaraiosis or superficial siderosis was not related to pretreatment with statins. Microbleeds were more frequent in patients with prior intracerebral hemorrhage (OR 9.7, 95\% Cl 1.06-90.9) and in those pre-treated antiplatelets (OR 1.66, 95\% Cl 1.09-2.53). Prior treatment with statins was not associated with markers of bleeding-prone cerebral angiopathies in patients with cardioembolic stroke. Therefore, previous statin treatment should not influence the decision to initiate or withhold oral anticoagulation if these neuroimaging markers are detected.

${ }^{1}$ Department of Neurology, Hospital de la Santa Creu i Sant Pau, Biomedical Research Institute, Barcelona, Spain. ${ }^{2}$ Department of Radiology, Hospital del Mar- Parc de Salut Mar, Barcelona, Spain. ${ }^{3}$ Hospital Germans Trias i Pujol, Unitat RM IDI, Badalona, Spain. ${ }^{4}$ Department of Epidemiology, Hospital de la Santa Creu i Sant Pau, Biomedical Research Institute, Barcelona, Spain. ${ }^{5}$ Department of Neurology, Hospital de Sant Joan Despí Moises Broggi, Sant Joan Despí, Spain. ${ }^{6}$ Department of Neurology, Hospital de León, León, Spain. ${ }^{7}$ Department of Neurology, Hospital San Pedro de Alcántara, Cáceres, Spain. ${ }^{8}$ Department of Neurology, Hospital Parc Tauli, Sabadell, Spain. ${ }^{9}$ Department of Neurology, Hospital Son Espases, Palma de Mallorca, Spain. ${ }^{10}$ Department of Neurology, Hospital de Basurto, Bilbao, Spain. ${ }^{11}$ Department of Neurology, Hospital de Valladolid, Valladolid, Spain. ${ }^{12}$ Department of Neurology, Hospital de Burgos, Burgos, Spain. ${ }^{13}$ Department of Neurology, Hospital de Granollers, Granollers, Spain. ${ }^{14}$ Department of Neurology, Hospital del Mar, Barcelona, Spain. ${ }^{15}$ Department of Neurology, Hospital de Navarra, Pamplona, Spain. ${ }^{16}$ Department of Neurology, Hospital Universitario La Paz, Instituto de Investigación IdiPaz, Madrid, Spain. ${ }^{17}$ Department of Neurology, Hospital Ramón y Cajal, Madrid, Spain. ${ }^{18}$ Department of Neurology, Hospital Clínic, Barcelona, Spain. ${ }^{19}$ Department of Neurology, Hospital Virgen de las Nieves, Granada, Spain. ${ }^{20}$ Department of Neurology, Hospital Germans Trias i Pujol, Badalona, Spain. ${ }^{21}$ Department of Neurology, Hospital Verge de la Cinta, Tortosa, Spain. ${ }^{22}$ Department of Neurology, Hospital de Donostia, Donostia, Spain. ${ }^{23}$ Department of Neurology, Hospital de Mataró, Mataró, Spain. ${ }^{24}$ Department of Neurology, Hospital Santiago de Compostela, Santiago de Compostela, Spain. ${ }^{25}$ Department of Neurology, Hospital de Albacete, Albacete, Spain. ${ }^{26}$ Department of Neurology, Hospital de La Rioja, Logroño, Spain. ${ }^{27}$ Department of Neurology, Centro Médico Asturias, Oviedo, Spain. ${ }^{28}$ Department of Neurology, Hospital Arnau de Vilanova, Lleida, Spain. ${ }^{29}$ Department of Neurology, Arcispedale Santa Maria Nuova IRCCS, Reggio Emilia, Italy. ${ }^{30}$ Port d'Informació Científica, Institut de Física d'Altes Energies, Campus UAB, Cerdanyola del Vallès, Spain. Correspondence and requests for materials should be addressed to J.M.-F. (email: jmarti@santpau.cat) 
It is common to prescribe statins for old patients. In a systematic review, up to $48 \%$ of patients who had an ischemic stroke were receiving statins prior to the stroke ${ }^{1}$. Statins are prescribed to prevent untoward vascular events, including ischemic stroke ${ }^{2}$. However, statins may increase the risk of intracerebral hemorrhage (ICH), due to the lowering of cholesterol levels and their antithrombotic properties ${ }^{3}$. Also, it is controversial whether or not statin therapy is linked to cerebral amyloid angiopathy (CAA) by increasing the likelihood of microbleeds (MB) and lobar hemorrhages ${ }^{4-10}$. Finally, the influence of the specific statin compound, the statin dose and the statin potency on the hemorrhagic risk has not been evaluated.

The fear of major bleeding, including intracranial hemorrhage, is the main reason why oral anticoagulants (OA) are underused in the elderly. Thus, the study of factors that contribute to the risk of hemorrhagic complications in long-term anticoagulation candidates is of enormous importance. It is known that cerebral hemorrhagic-prone amyloid and hypertensive angiopathies increase the risk of ICH in older patients. Additionally, drugs with antithrombotic properties might increase ICH risk in patients with these angiopathies $^{11-13}$. Consequently, the combination of statins and antiplatelet or OA drugs, might lead to excessive hemorrhagiing.

In a prospective study, our aim was to investigate whether prior statin therapy increases the risk of $\mathrm{MB}$, cortical superficial siderosis (SS) and leukoaraiosis (LK) in patients with cardioembolic stroke who did not take OA and who were candidates to begin indefinite treatment with OA. Our hope was that our study would provide guidance regarding the use of statins in patients who have suffered a cardioembolic stroke and in whom OA are to be prescribed.

\section{Material and Methods}

We studied patients with transient ischemic attack (TIA) or cerebral infarct attributed to cardiac embolism after a complete etiologic work-up and who were considered candidates for indefinite OA treatment. For most patients, atrial fibrillation (AF) was the embolic source, but patients with other high-risk cardioembolic sources were also permitted. All of the patients were naïve to OA. The patients were participants in the HERO study. The HERO study (risk of intracranial HEmorrhage predicted by Resonance in patients receiving Oral anticoagulants, ClinicalTrials.gov Identifier: NCT02238470), a currently ongoing multicenter observational study. Briefly, the aim of the study is to assess whether the risk of intracranial hemorrhage (intracerebral and extracerebral) in patients with cardioembolic stroke who start a long-term treatment with OA is influenced by the detection of surrogate markers of hemorrhagic-prone angiopathies in the MRI. All methods were carried out in accordance with relevant guidelines and regulations. All experimental protocols were approved by the Ethics Committee at Hospital de la Santa Creu i Sant Pau and also at each center. Informed written consent was required for study participation. The patient or a legal representative signed the written consent to participate.

Patients. Inclusion criteria were: 1) age equal or above 65 years; 2) TIA or acute ischemic infarct; 3) the patient is considered a good candidate to receive long-term OA for the secondary prevention of ischemic stroke; 4 ) the patient is a new user of any OA (vitamin $\mathrm{K}$ antagonist or direct OA); 5) the consent to participate is signed before performing MR; 6) MR is performed within 1 month of the index ischemic stroke.

Exclusion criteria were: 1) Primary prevention of cardiac embolism; 2) Indication of OA other than the prevention of cardiac embolism; 3) Absolute contraindication to OA treatment; 4) Uncontrolled high blood pressure and hypertensive crisis; 5) Dementia; 6) Life expectancy less than 1 year; 7) Any contraindication to perform an MR.

The following clinical variables were recorded for each patient: 1) Demographic data (age and gender); 2) Traditional vascular risk factors (previous cerebral infarct and TIA, previous intracerebral hemorrhage, hypertension, diabetes mellitus, hypercholesterolemia, hypertriglicerydemia, smoking, alcohol abuse, obesity), chronic renal insufficiency, ischemic heart disease, peripheral vascular disease, atrial fibrillation, valvular heart disease, other cardioembolic sources; 3) Pre-treatment with antiplatelet agents; 4) The $\mathrm{CHA}_{2} \mathrm{DS}_{2}$ Vasc score and the HASBLED score; 5) Statin status: pre-treatment or no pre-treatment with any statin. In patients receiving statins, the specific statin compound and its dose were recorded. In addition, the intensity of the statin therapy was classified into 3 types (low, medium or high) according to accepted guidelines ${ }^{14}$.

Neuroradiological evaluation. MR scans were performed according to standard protocols at each site and at least included a T2*-weighted gradient-recalled echo (T2*-GRE) and/or susceptibility-weighted imaging (SWI) for the assessment of MB and cortical SS, and fluid-attenuated inversion recovery (FLAIR) for the assessment of LK. Two neuroradiologists, who were blind to clinical data including the statin status, evaluated the MR scans. The following abnormalities were noted: 1) MB, defined as rounded small lesions of less than $10 \mathrm{~mm}$, as evidenced in echo-gradient or susceptibility images after ruling out MB mimics. In addition, its distribution (hemispheric -cortical, deep, or both) were recorded; 2) LK, defined as deep and periventricular white matter hyperintense lesions detected on T2 or FLAIR sequences. Its severity was quantified by the Fazekas' scale ${ }^{15} ; 3$ ) Cortical SS, defined as signal loss on T2*-GRE and SWI sequences in a curvilinear pattern following the gyral cortical surface ${ }^{16}$.

Statistical analysis. We compared demographic, clinical and neuroimaging variables of patients with or without prior treatment with statins. The results are expressed as percentages for categorical variables and as mean and standard deviation (SD) for the continuous variables. The proportions were compared using the Chi-square $\left(\chi^{2}\right)$ test or the Fisher exact test when it was required, while the mean and SD of continuous variables between groups were compared by the Student's t-test. Median values for $\mathrm{CHA}_{2} \mathrm{DS}_{2}$ Vasc and HASBLED scores were compared by the Mann-Whitney $U$ test. We repeated the bivariate analyses by dividing the patients into four groups (no prior treatment, and low, medium or high prior intensity treatment). We also analyzed separately also 


\begin{tabular}{|l|l|l|}
\hline Name brand and daily dose of statin & Number (\%) & Intensity \\
\hline Simvastatin & $80(17 \%)$ & \\
\hline $10 \mathrm{mg}$ & 15 & Low \\
\hline $20 \mathrm{mg}$ & 46 & Medium \\
\hline $40 \mathrm{mg}$ & 15 & Medium \\
\hline Dose unknown & 4 & N/A \\
\hline Atorvastatin & $76(16.2 \%)$ & \\
\hline $10 \mathrm{mg}$ & 12 & Medium \\
\hline $20 \mathrm{mg}$ & 32 & Medium \\
\hline $40 \mathrm{mg}$ & 21 & High \\
\hline $80 \mathrm{mg}$ & 9 & High \\
\hline Dose unknown & 2 & N/A \\
\hline Pravastatin & $10(2.1 \%)$ & \\
\hline $10 \mathrm{mg}$ & 1 & Low \\
\hline $20 \mathrm{mg}$ & 7 & Low \\
\hline $40 \mathrm{mg}$ & 2 & Medium \\
\hline Pitavastatin & $1(0.2 \%)$ & \\
\hline $1 \mathrm{mg}$ & 1 & Low \\
\hline Lovastatin & $4(0.8 \%)$ & \\
\hline $10 \mathrm{mg}$ & 1 & Low \\
\hline $20 \mathrm{mg}$ & 3 & Low \\
\hline Rosuvastatin & $10(2.1 \%)$ & \\
\hline $5 \mathrm{mg}$ & 2 & Medium \\
\hline $10 \mathrm{mg}$ & 6 & \\
\hline $20 \mathrm{mg}$ & 2 & \\
\hline Fluvastatin & $3(0.6 \%)$ & \\
\hline $80 \mathrm{mg}$ & 3 & \\
\hline Treatment with statin with specific \\
compound unknown & $9(2)$ & \\
\hline
\end{tabular}

Table 1. Characteristics of the statin therapy: statin and daily dose. Values are given in number and (percentage).

each of the specific statin compounds. Finally, we performed a logistic regression analysis, to investigate the variables associated with MB. This was a forward stepwise analysis with those variables with a $\mathrm{p} \leq 0.1$ in the bivariate analyses. We adjusted the final model for age and potential confounders.

Data availability. The datasets generated during and/or analysed during the current study are available from the corresponding author on reasonable request.

\section{Results}

We studied 470 patients. Their mean age was $77.5 \pm 6.4$ years and $43.7 \%$ of them were men. The reason to prescribe OA was AF for 418 patients, atrial flutter for 15, valve disease for 9, dilated myocardiopathy for 6 and other reasons for 23. For the substudy of statin compound and dosage we excluded 15 patients in whom this information was unknown. A total of 193 (41.1\%) patients were receiving statins prior to admission and the specific statin that was administered and its dose details are listed in Table 1 . The intensity of statin treatment of the 178 patients with known dose was as follows: 28 patients (15.7\% of the statin group) received a low dose, 118 (66.2\% of the statin group) received a medium dose and 32 patients ( $17.9 \%$ of the statin group) received a high dose. Most of the patients who received statins prior to their stroke were treated with atorvastatin $(n=76)$ or $\operatorname{simvastatin}(n=80)$.

Table 2 shows the demographic data of patients with and without prior statin treatment. As expected, patients in the statin group had a higher frequency of vascular risk factors: hypercholesterolemia, hypertryglyceridemia, diabetes, hypertension and of previous ischemic events in the peripheral, coronary or cerebral areas. Also, they had higher scores on the $\mathrm{CHA}_{2} \mathrm{DS}_{2}$ Vasc and HAS-BLED scores and a higher frequency of concomitant treatment with antiplatelet agents.

MR images were obtained with a $1 \mathrm{~T}$ file strength in $40(8.5 \%)$ patients, $1.5 \mathrm{~T}$ in $365(77.8 \%)$, and $3 \mathrm{~T}$ in 64 (13.6\%) patients. T2*-GRE sequences only were obtained in 187 patients, SWI only in 4 and both in 278 . MB were detected in $140(29.7 \%)$ patients. The distribution was lobar in 55\%, deep in $30 \%$ and both in $15 \%$ of the patients. A total of 66 of them ( $47 \%$ of the MB group and $14 \%$ of the total patients) had more than one MB, and 8 ( $6 \%$ of the MB group and $1.7 \%$ of the total sample) more than $10 \mathrm{MBs}$. There were no differences in the presence of $\mathrm{MB}$ or in the $\mathrm{MB}$ burden between those patients with and without statin treatment. Moreover, the distribution of $\mathrm{MB}$ was not different between patients who were pre-treated or those who were not pre-treated with statins (Table 2). LK was detected in $388(82.5 \%)$ of the patients and was severe in $19.8 \%$. Cortical SS was detected in $20(4.3 \%)$ patients. Likewise, the presence of cortical SS and the presence and burden of LK were equivalent in 


\begin{tabular}{|c|c|c|c|}
\hline Variable & $\begin{array}{l}\text { Prior statin } \\
\text { treatment } \\
(n=193)\end{array}$ & $\begin{array}{l}\text { No prior statin } \\
\text { treatment } \\
(\mathbf{n}=277)\end{array}$ & p value \\
\hline Age $(y)$ & $78.01 \pm 5.5$ & $77.2 \pm 7$ & 0.21 \\
\hline Sex distribution (\% men) & 44 & 43.3 & 0.88 \\
\hline Type of stroke (\% cerebral infarct) & 84.9 & 88.4 & 0.17 \\
\hline Previous cerebral infarct & 26.9 & 19.1 & 0.05 \\
\hline Previous Transient Ischemic Attack & 14 & 5.4 & 0.002 \\
\hline Previous Intracerebral Hemorrhage & 0.5 & 1.4 & 0.34 \\
\hline Hypertension & 75.6 & 65.3 & 0.007 \\
\hline Diabetes Mellitus & 26.9 & 13 & $<0.001$ \\
\hline Hypercholesterolemia & 79.8 & 19.5 & $<0.001$ \\
\hline Hypertriglyceridemia & 10.4 & 4 & 0.002 \\
\hline Smoking & 7.8 & 7.6 & 0.65 \\
\hline Alcohol abuse & 3.6 & 4 & 0.76 \\
\hline Obesity & 21.8 & 15.5 & 0.13 \\
\hline Chronic renal failure & 7.8 & 6.1 & 0.49 \\
\hline Ischemic heart disease & 24.4 & 5.4 & $<0.001$ \\
\hline Peripheral vascular disease & 8.3 & 1.4 & 0.001 \\
\hline Previous Atrial Fibrillation & 48.7 & 48.4 & 0.36 \\
\hline Valvular heart disease & 3.1 & 4 & 0.59 \\
\hline Other cardioembolic sources & 4.1 & 3.6 & 0.62 \\
\hline Prior treatment with anti platelets & 61.1 & 30.7 & $<0.001$ \\
\hline $\mathrm{CHA}_{2} \mathrm{DS}_{2}$ Vasc score [median (IQ range)] & $6(5-6)$ & $5(4-6)$ & $<0.001$ \\
\hline HASBLED score [median (IQ range)] & $2(2-2)$ & $2(2-2)$ & 0.007 \\
\hline Microbleed (yes or not) & 30.6 & 29.2 & 0.76 \\
\hline \multicolumn{4}{|l|}{ Microbleed burden } \\
\hline 0 & 69.4 & 70.4 & \\
\hline 1 & 18.7 & 13.7 & 0.24 \\
\hline$>1$ & 11.9 & 15.5 & \\
\hline \multicolumn{4}{|l|}{ Microbleed distribution hemispheric } \\
\hline lobar & 12 & 6.9 & \\
\hline deep & 13.5 & 18.4 & 0.14 \\
\hline both & 5.2 & 4 & \\
\hline Cortical siderosis (yes or not) & 2.7 & 5.5 & 0.17 \\
\hline \multicolumn{4}{|l|}{ Leukoaraiosis } \\
\hline 0 & 18 & 17.1 & \\
\hline 1 & 34.9 & 37.8 & 0.17 \\
\hline 2 & 22.8 & 28.4 & \\
\hline 3 & 24.3 & \begin{tabular}{|l|}
16.7 \\
\end{tabular} & \\
\hline
\end{tabular}

Table 2. Demographic data, vascular risk factors, risk scores, and MR results in patients with or without prior statin therapy. Values are given in percentage, unless specified otherwise.

patients with and without previous statin treatment. As shown in Table 3, the presence or burden of MB, presence and degree of LK and presence of cortical SS according to the statin intensity also showed no differences among statin intensity groups. The results were equivalent for treatment with atorvastatin and simvastatin.

Finally, we performed a logistic regression analysis to predict the presence of MB. From a first model including those variables that in the bivariate analyses (Table 4 ) showed an association $(p \leq 0.1)$ with the risk of finding MB (previous cerebral infarct, previous intracerebral hemorrhage, smoking, alcohol abuse, obesity, hypertension and previous antiplatelet treatment) we conducted a forward stepwise selection method (Table 5). The model was adjusted by known potential contributors. When adjusting the final model for age, prior ICH (OR 9.70, 95\% CI 1.066-90.9) and prior antiplatelet (OR 1.66, 95\% CI 1.09-2.53) remained the only variables associated with the risk of MB. Remarkably, the inclusion of the variable prior statin therapy did not alter the final model coefficients and did not show any interaction with the variables included.

\section{Discussion}

Our multicenter observational study of patients with cardioembolic stroke indicated that patients who received statins prior to stroke did not show a higher frequency or burden of surrogate MR markers for bleeding-prone angiopathies than patients who did not receive statins. This suggests that statins do not interact with hemorrhagic-prone small-vessel angiopathies. Also, we found that the intensity of the statin treatment was not associated with the presence or the burden of MB, LK or cortical SS. 


\begin{tabular}{|c|c|c|c|c|}
\hline & $\begin{array}{l}\text { Low intensity } \\
(\mathbf{n}=28)\end{array}$ & $\begin{array}{l}\text { Medium intensity } \\
(\mathrm{n}=118)\end{array}$ & $\begin{array}{l}\text { High intensity } \\
\mathrm{n}=32\end{array}$ & p value \\
\hline MB & $12(42.9)$ & $33(28)$ & $9(28.1)$ & 0.29 \\
\hline \multicolumn{5}{|l|}{ MB burden } \\
\hline 0 & $16(57.1)$ & $85(72)$ & $23(71.9)$ & \\
\hline 1 & $7(25)$ & $20(16.9)$ & $4(12.5)$ & 0.53 \\
\hline$>1$ & $5(17.9)$ & $13(11)$ & $5(15.6)$ & \\
\hline \multicolumn{5}{|c|}{ MB distribution $(\mathrm{n}=54$ with 1 or more $\mathrm{MB})$} \\
\hline Lobar & $5(41.7)$ & $14(42.4)$ & $3(33.3)$ & \\
\hline Deep & $5(41.7)$ & $14(42.4)$ & $3(33.3)$ & 0.81 \\
\hline Both & $2(16.7)$ & $5(15.2)$ & $3(33.3)$ & \\
\hline Cortical superficial siderosis & $1(4)$ & $4(3.6)$ & $0(0)$ & 0.54 \\
\hline \multicolumn{5}{|l|}{ Leukoaraiosis score } \\
\hline 0 & $5(18.5)$ & $18(15.7)$ & $7(21.9)$ & \\
\hline 1 & $11(40.7)$ & $42(36.5)$ & $7(21.9)$ & 0.66 \\
\hline 2 & $6(22.2)$ & $27(23.5)$ & $7(21.9)$ & \\
\hline 3 & $5(18.5)$ & $28(24.3)$ & $11(34.4)$ & \\
\hline
\end{tabular}

Table 3. Surrogate markers of angiopathy according to the intensity of statin pre-treatment. Values are given in number and percentage.

\begin{tabular}{|l|l|l|l|}
\hline Variable & $\begin{array}{l}\text { MB yes } \\
(\mathbf{n}=\mathbf{1 4 0})\end{array}$ & $\begin{array}{l}\text { MB no } \\
(\mathbf{n = 3 3 0})\end{array}$ & p value \\
\hline Age (y) & $78(6.9)$ & $77.3(6.2)$ & 0.34 \\
\hline Gender distribution (\% men) & 45 & 43 & 0.88 \\
\hline Previous cerebral infarct & 25.7 & 20.9 & 0.058 \\
\hline Previous Intracerebral Hemorrhage & 2.9 & 0.3 & 0.006 \\
\hline Hypertension & 68.5 & 72.1 & 0.097 \\
\hline Diabetes Mellitus & 19.3 & 18.5 & 0.25 \\
\hline Hypercholesterolemia & 42.9 & 44.8 & 0.211 \\
\hline Hypertriglyceridemia & 7.1 & 6.4 & 0.34 \\
\hline Smoking & 10 & 6.7 & 0.083 \\
\hline Alcohol abuse & 1.4 & 4.8 & 0.067 \\
\hline Obesity & 19.3 & 17.6 & 0.078 \\
\hline Chronic renal failure & 8.6 & 6.1 & 0.11 \\
\hline Ischemic heart disease & 12.9 & 13.3 & 0.211 \\
\hline Peripheral vascular disease & 5.7 & 3.6 & 0.203 \\
\hline Previous Atrial Fibrillation & 51.4 & 47.3 & 0.175 \\
\hline Prior treatment with antiplatelets & 50.7 & 40 & 0.015 \\
\hline Prior treatment with statins & 42.1 & 40.6 & 0.760 \\
\hline CHA ${ }_{2}$ DS ${ }_{2}$ Vasc score [median (IQ range)] & $5(4-6)$ & $5(4-6)$ & 0.96 \\
\hline HASBLED score [median (IQ range)] & $2(2-2)$ & $2(2-2)$ & 0.324 \\
\hline & & & \\
\hline
\end{tabular}

Table 4. Bivariate analyses of variables in patients with and without MB. Values are given in percentage, unless specified otherwise.

The reason why there is an association between statin treatment and an increased risk of intracranial hemorrhage (ICH) is not fully understood. Some previous studies suggested that statin therapy increases the risk of ICH, specially in patients with a history of cerebrovascular disease ${ }^{2,17}$. In patients treated with statins there is an increase in symptomatic intracerebral hemorrhage after intravenous or endovascular reperfussion therapies $^{18-21}$. However, this increased risk of ICH after exposure to statins has not been confirmed by other trials or meta-analyse $\mathrm{s}^{22-25}$. Some explanations have been presented for the increased hemorrhagic risk associated with statin therapy. First, statin therapy is antithrombotic ${ }^{3}$, and it enhances anticoagulant and antiplatelet effects as well as an enhanced fibrinolysis. Second, statin therapy lowers cholesterol levels. Cholesterol levels are inversely associated with risk of hemorrhagic stroke ${ }^{26-29}$. It is important to note that LDL levels at baseline and during treatment were not related to haemorrhage risk in the SPARCL ${ }^{30}$ and PCSK9 inhibitors trials ${ }^{31,32}$.

Whether statin increases the risk of microhemorrhages, evidenced by $\mathrm{MB}$, or other hemorrhagic-prone angiopathy markers is unknown. MB is a marker of hypertensive and amyloid angiopathies, which are the most frequent etiologies in patients with spontaneous $\mathrm{ICH}^{33,34}$. By analogy with $\mathrm{OA}$ and antiplatelet treatment, it is plausible that the antithrombotic properties of statins might increase the bleeding risk of macro as well as 


\begin{tabular}{|l|l|l|l|l|}
\hline & Variables & OR & 95\% IC & p \\
\hline \multirow{2}{*}{ Model 1* } & Previous ICH & 9.708 & $1.057-90.909$ & 0.044 \\
\cline { 2 - 5 } & Previous antiplatelet & 1.694 & $1.116-2.570$ & 0.013 \\
\hline \multirow{2}{*}{ Model $2^{\dagger}$} & Previous ICH & 9.708 & $1.066-90.909$ & 0.044 \\
\cline { 2 - 5 } & Previous antiplatelet & 1.669 & $1.098-2.538$ & 0.016 \\
\hline
\end{tabular}

Table 5. Logistic regresion analysis to predict the presence of MB. *(Adjusted for prior cerebral infarct, smoking, alcohol abuse, obesity and arterial hypertension). ${ }^{\dagger}$ (Adjusted for age).

microhemorrhages. Whereas some authors advise to avoid statin therapy after an ICH, whether this is prudent also when micro- instead of macrohemorrhages are detected is unclear ${ }^{35}$. In our multicenter study of 470 patients, $41 \%$ of our patients were taking statins before stroke, but we were unable to find an association between statin treatment and MB. About $30 \%$ of our patients exhibited at least $1 \mathrm{MB}, 4.5 \%$ cortical SS and $80 \%$ some degree of LK. We restricted our study to patients with cardioembolic stroke because, although they constitute the subtype of ischemic stroke with less $\mathrm{MB}$, they often are prescribed $\mathrm{OA}^{36}$. We included only patients who were new users of OA to avoid any possible influence of previous treatment with OA in the development of these markers ${ }^{37}$. Moreover, although the concomitant treatment with antiplatelets was related in our study to the presence of MB, the use of statins was not, even after including this variable in our logistic regression analysis.

We evaluated different intensities of statin therapy and found that there was no effect on MB, LK, and cortical $\mathrm{SS}^{14}$. Finally, due to the reported differences and even opposite effects among statin compounds we investigated the different effects of the two most prescribed statin brands (simvastatin and atorvastatin) ${ }^{38}$. We did not find any influence on the presence or burden of hemorrhagic-prone markers.

Our results are in accordance with two studies ${ }^{6,7}$. One retrospective study of patients with ischemic stroke was unable to find any association between statin treatment and $\mathrm{MB}$ in any location ${ }^{6}$. Importantly, the etiologic stroke subtype was not analyzed in this study. A 2-year follow-up study of patients with stroke of any etiology included in the VITATOPS trial demonstrated also that statin treatment had no effect on incident lacunes or incident $\mathrm{MB}^{7}$.

Conversely, other studies suggest that statins might cause cerebral amyloid angiopathy (CAA) or MB, and may therefore trigger bleeding complications, but it is unknown whether statin treatment or low colesterol levels increase the amount of $\mathrm{MB}^{10}$. A recent study reported that the protective effect of serum cholesterol against intracerebral hemorrhage was reduced by statins but only in lobar regions ${ }^{8}$. The Framingham Heart Study indicated that statin increased the risk of lobar and mixed location $\mathrm{MB}^{4}$. A multivariate analysis of patients with or without stroke found an association between very low total cholesterol levels and MB burden ${ }^{9}$. Finally, a retrospective multivariate analysis of patients with intracerebral hemorrhage found that patients who were treated with statins had almost twice as many cortical MB compared to patients not treated with statins ${ }^{5}$. Unfortunately, these studies are not completely comparable with our study since they include patients without stroke or with hemorrhagic stroke $e^{4,5,8,9}$. Because we found that lobar MB and cortical SS are not related to previous statin therapy, we propose that statins do noy play a role in the development of intracranial angiopathies. Further studies, including genetic analyses, are needed to clarify this point ${ }^{10,39}$. There may be a subset of patients with certain genetic traits (such as $\varepsilon 2$ and $\varepsilon 4$ alleles of the Apolipoprotein E gene) that may confer susceptibility to brain microbleeds after statin exposure.

Our study has some limitations. First, we cannot be sure that the prescription of OA toghether with statins during secondary prevention is safe. The results of ongoing studies may help clarifying whether statins are safe in patients who have suffered a cardioembolic ischemic stroke and receive OA. No interaction between statin treatment and antithrombotic treatments were detected in the SPARCL study, and a small randomized trial found no increase in the risk of bleeding in patients who received statin and $\mathrm{OA}^{30,40}$. We have not provided information on statin adherence and in duration of previous treatment. It is possible that harmful effects of statin, if any, only appear after a long period of treatment. Moreover, the non-randomized use of statins in our study means that statin use is confounded by the indication for statin prescription. We did not measure colesterol levels and therefore cannot affirm or deny that they are associated with the markers. Statin treatment with high doses, a surrogate for lower levels of cholesterol, argues against the relevance of colesterol levels in the development of MB. We measured the radiological markers from different centers that used different imaging protocols and different MR apparatus of different field strength, and this could affect our results. Finally, the statistical power may not be sufficient to analyse the impact of statins on a number of surrogate markers.

\section{Summary}

Statin therapy does not appear to increase the risk of MB, cortical SS or LK in patients with cardioembolic stroke who have not received OA. Our results suggest that the potential mechanism of statin-associated ICH, is not mediated by an increase in MB, cortical SS or LK. However, further studies including follow-up data after OA are needed. In the absence of definitive results, it is prudent to not discontinue or withhold statin treatment when $\mathrm{MB}$, cortical SS or LK are detected.

\section{References}

1. Ní Chróinín, D. et al. Statin therapy and outcome after ischemic stroke: systematic review and meta-analysis of observational studies and randomized trials. Stroke. 44, 448-456 (2013)

2. Amarenco, P. et al. High-dose atorvastatin after stroke or transient ischemic attack. N Engl J Med. 355, 549-559 (2006).

3. Violi, F., Calvieri, C., Ferro, D. \& Pignatelli, P. Statins as antithrombotic drugs. Circulation. 127, 251-257 (2013). 
4. Romero, J. R. et al. Risk factors, stroke prevention treatments, and prevalence of cerebral microbleeds in the Framingham Heart Study. Stroke. 45, 1492-1494 (2014).

5. Haussen, D. C., Henninger, N., Kumar, S. \& Selim, M. Statin use and microbleeds in patients with spontaneous intracerebral hemorrhage. Stroke. 43, 2677-2681 (2012).

6. Day, J. S. et al. Previous statin use is not associated with an increased prevalence or degree of gradient-echo lesions in patients with acute ischemic stroke or transient ischemic attack. Stroke. 42, 354-358 (2011).

7. Xiong, Y. et al. Prestroke statins, progression of white matter hyperintensities, and cognitive decline in stroke patients with confluent white matter hyperintensities. Neurotherapeutics. 11, 606-611 (2014).

8. Pezzini, A. et al. Serum cholesterol levels, HMG-CoA reductase inhibitors and the risk of intracerebral haemorrhage. The Multicenter Study on Cerebral Haemorrhage in Italy (MUCH-Italy). J Neurol Neurosurg Psychiatry. 87, 924-929 (2016).

9. Lee, S. H. et al. Low concentration of serum total cholesterol is associated with multifocal signal loss lesions on gradient-echo magnetic resonance imaging: analysis of risk factors for multifocal signal loss lesions. Stroke 33, 2845-2849 (2002).

10. Woo, D. et al. Apolipoprotein E, statins, and risk of intracerebral hemorrhage. Stroke. 44, 3013-3017 (2013).

11. Gregoire, S. M. et al. Brain microbleeds as a potential risk factor for antiplatelet-related intracerebral haemorrhage: hospital-based, case-control study. J Neurol Neurosurg Psychiatry. 81, 679-684 (2010).

12. van Etten, E. S. et al. Incidence of symptomatic hemorrhage in patients with lobar microbleeds. Stroke. 45, 2280-2285 (2014).

13. Lee, S. H., Ryu, W. S. \& Roh, J. K. Cerebral microbleeds are a risk factor for warfarin-related intracerebral hemorrhage. Neurology. 72, 171-176 (2009).

14. Stone, N. J. et al. ACC/AHA guideline on the treatment of blood cholesterol to reduce atherosclerotic cardiovascular risk in adults: a report of the American College of Cardiology/American Heart Association Task Force on Practice Guidelines. J Am Coll Cardiol 63(25 Pt B), 2889-2934 (2014).

15. Fazekas, F., Chawluk, J. B., Alavi, A., Hurtig, H. I. \& Zimmerman, R. A. MR signal abnormalities at 1.5 T in Alzheimer's dementia and normal aging. AJR Am J Roentgenol. 149, 351-356 (1987).

16. Charidimou, A. et al. Cortical superficial siderosis: detection and clinical significance in cerebral amyloid angiopathy and related conditions. Brain. 138, 2126-2139 (2015).

17. Vergouwen, M. D., de Haan, R. J., Vermeulen, M. \& Roos, Y. B. Statin treatment and the occurrence of hemorrhagic stroke in patients with a history of cerebrovascular disease. Stroke. 39, 497-502 (2008).

18. Scheitz, J. F. et al. Dose-related effects of statins on symptomatic intracerebral hemorrhage and outcome after thrombolysis for ischemic stroke. Stroke. 45, 509-514 (2014).

19. Martinez-Ramirez, S. et al. Statin pretreatment may increase the risk of symptomatic intracranial haemorrhage in thrombolysis for ischemic stroke: results from a case-control study and a meta-analysis. J Neurol. 259, 111-118 (2012).

20. Bang, O. Y. et al. Cholesterol level and symptomatic hemorrhagic transformation after ischemic stroke thrombolysis. Neurology. 68, 737-742 (2007).

21. Meier, N. et al. Prior statin use, intracranial hemorrhage, and outcome after intra-arterial thrombolysis for acute ischemic stroke. Stroke. 40, 1729-1737 (2009).

22. Collins, R., Armitage, J., Parish, S., Sleight, P. \& Peto, R. Heart Protection Study Collaborative G. Effects of cholesterol-lowering with simvastatin on stroke and other major vascular events in 20536 people with cerebrovascular disease or other high-risk conditions. Lancet. 363, 757-767 (2004).

23. Cholesterol Treatment Trialists' (CTT) Collaboration. et al. Efficacy and safety of more intensive lowering of LDL cholesterol: a meta-analysis of data from 170000 participants in 26 randomised trials. Lancet, 376:1670-1681 (2010).

24. Hackam, D. G. et al. Statins and intracerebral hemorrhage: collaborative systematic review and meta-analysis. Circulation. 124, 2233-2242 (2011).

25. McKinney, J. S. \& Kostis, W. J. Statin therapy and the risk of intracerebral hemorrhage: a meta-analysis of 31 randomized controlled trials. Stroke. 43, 2149-2156 (2012).

26. Lauer, A., Greenberg, S. M. \& Gurol, M. E. Statins in Intracerebral Hemorrhage. Curr Atheroscler Rep. 17, 46 (2015).

27. Wang, X., Dong, Y., Qi, X., Huang, C. \& Hou, L. Cholesterol levels and risk of hemorrhagic stroke: a systematic review and metaanalysis. Stroke. 44, 1833-1839 (2013).

28. Woo, D. et al. Hypercholesterolemia, HMG-CoA reductase inhibitors, and risk of intracerebral hemorrhage: a case-control study. Stroke. 35, 1360-1364 (2004).

29. Sikora Newsome, A. et al. The Role of Statin Therapy in Hemorrhagic Stroke. Pharmacotherapy. 35, 1152-1163 (2015).

30. Goldstein, L. B. et al. Hemorrhagic stroke in the stroke prevention by aggressive reduction in cholesterol levels study. Neurology. 70, 2364-2370 (2008).

31. Robinson, J. G. et al. Efficacy and safety of alirocumab in reducing lipids and cardiovascular events. N Engl J Med. 372, 1489-1499 (2015).

32. Sabatine, M. S. et al. Efficacy and safety of evolocumab in reducing lipids and cardiovascular events. N Engl J Med. 372, 1500-1509 (2015).

33. Fazekas, F. et al. Histopathologic analysis of foci of signal loss on gradient-echo T2*-weighted MR images in patients with spontaneous intracerebral hemorrhage: evidence of microangiopathy-related microbleeds. AJNR Am J Neuroradiol. 20, 637-642 (1999).

34. Martí-Fàbregas, J. et al. The H-ATOMIC Criteria for the Etiologic Classification of Patients with Intracerebral Hemorrhage. PLoS One. 11, e0156992 (2016).

35. Westover, M. B., Bianchi, M. T., Eckman, M. H. \& Greenberg, S. M. Statin use following intracerebral hemorrhage: a decision analysis. Arch Neurol. 68, 573-579 (2011).

36. Cordonnier, C., Al-Shahi Salman, R. \& Wardlaw, J. Spontaneous brain microbleeds: systematic review, subgroup analyses and standards for study design and reporting. Brain. 130, 1988-2003 (2007).

37. Lovelock, C. E., Cordonnier, C., Naka, H., Al-Shahi Salman, R. \& Sudlow, C. L. Edinburgh Stroke Study Group, et al. Antithrombotic drug use, cerebral microbleeds, and intracerebral hemorrhage: a systematic review of published and unpublished studies. Stroke. 41, $1222-1228$ (2010).

38. Palinski, W. \& Napoli, C. Unraveling pleiotropic effects of statins on plaque rupture. Arterioscler Thromb Vasc Biol 22, 1745-1750 (2002).

39. Banach, M. et al. Intensive LDL-cholesterol lowering therapy and neurocognitive function. Pharmacol Ther. 170, 181-191 (2017).

40. Enajat, M. et al. Safety of the combination of intensive cholesterol-lowering therapy with oral anticoagulation medication in elderly patients with atrial fibrillation: a randomized, double-blind, placebo-controlled study. Drugs Aging. 26, 585-593 (2009).

\section{Acknowledgements}

This study was funded by the Spanish Ministery of Health - Instituto Carlos III, Redes Temáticas de Investigación Cooperativa (INVICTUS PLUS RD16/0019/0010) and Fondo de Investigaciones Sanitarias FI12/00296. The study also received funding from FEDER (Fondo Europeo de Desarrollo Regional). The study was done with the aid of the Stroke Project, Cerebrovascular Diseases Group of the Spanish Neurological Society. 


\section{Author Contributions}

Joan Martí-Fàbregas: Design and conceptualization of the study, analysis and interpretation of the data, drafting and revising the manuscript. Santiago Medrano-Martorell: analysis and interpretation of the data, drafting and revising the manuscript. Elisa Merino: analysis and interpretation of the data, drafting and revising the manuscript. Luis Prats-Sánchez: Design and conceptualization of the study, analysis and interpretation of the data, drafting and revising the manuscript. Rebeca Marín: Data collection, Analysis or interpretation of the data, revising the manuscript. Raquel Delgado-Mederos: Data collection, Design and conceptualization of the study, analysis and interpretation of the data, drafting and revising the manuscript. Pol Camps-Renom: Data collection, Analysis and interpretation of the data, revising the manuscript. Alejandro Martínez-Domeño: Data collection, Analysis and interpretation of the data, revising the manuscript. Manuel Gómez-Choco: Data collection, Analysis and interpretation of the data, revising the manuscript. Lidia Lara: Data collection, Analysis and interpretation of the data, revising the manuscript. Ignacio Casado-Naranjo: Data collection, Analysis and interpretation of the data, revising the manuscript. David Cánovas: Data collection, Analysis or interpretation of the data, revising the manuscript. Maria José Torres: Data collection, Analysis and interpretation of the data, revising the manuscript. Marimar Freijo: Data collection, Analysis and interpretation of the data, revising the manuscript. Ana Calleja: Data collection, Analysis and interpretation of the data, revising the manuscript. Yolanda Bravo: Data collection, Analysis and interpretation of the data, revising the manuscript. Dolores Cocho: Data collection, Analysis and interpretation of the data, revising the manuscript. Ana Rodríguez-Campello: Data collection, Analysis and interpretation of the data, revising the manuscript. Beatriz Zandio: Data collection, Analysis and interpretation of the data, revising the manuscript. Blanca Fuentes: Data collection, Analysis and interpretation of the data, revising the manuscript. Alicia de Felipe: Data collection, Analysis and interpretation of the data, revising the manuscript. Laura Llull: Data collection, Analysis and interpretation of the data, revising the manuscript. José Maestre: Data collection, Analysis and interpretation of the data, revising the manuscript. María Hernández: Data collection, Analysis and interpretation of the data, revising the manuscript. Moisès Garcés: Data collection, Analysis and interpretation of the data, revising the manuscript. Ana Maria De Arce-Borda: Data collection, Analysis and interpretation of the data, revising the manuscript. Ernest Palomeras: Data collection, Analysis and interpretation of the data, revising the manuscript. Manuel Rodríguez-Yáñez: Data collection, Analysis and interpretation of the data, revising the manuscript. Inma Díaz-Maroto: Data collection, Analysis and interpretation of the data, revising the manuscript. Marta Serrano: Data collection, Analysis and interpretation of the data, revising the manuscript. Jéssica Fernández-Domínguez: Data collection, Analysis and interpretation of the data, revising the manuscript. Jordi Sanahuja: Data collection, Analysis and interpretation of the data, revising the manuscript. Francisco Purroy: Data collection, Analysis and interpretation of the data, revising the manuscript. Marialuisa Zedde: Data collection, Analysis and interpretation of the data, revising the manuscript. Jordi Delgado-Mengual: Data collection, Analysis and interpretation of the data, revising the manuscript. Ignasi Gich: Design and conceptualization of the study, analysis and interpretation of the data, drafting and revising the manuscript.

\section{Additional Information}

Competing Interests: The authors declare that they have no competing interests.

Publisher's note: Springer Nature remains neutral with regard to jurisdictional claims in published maps and institutional affiliations.

Open Access This article is licensed under a Creative Commons Attribution 4.0 International License, which permits use, sharing, adaptation, distribution and reproduction in any medium or format, as long as you give appropriate credit to the original author(s) and the source, provide a link to the Creative Commons license, and indicate if changes were made. The images or other third party material in this article are included in the article's Creative Commons license, unless indicated otherwise in a credit line to the material. If material is not included in the article's Creative Commons license and your intended use is not permitted by statutory regulation or exceeds the permitted use, you will need to obtain permission directly from the copyright holder. To view a copy of this license, visit http://creativecommons.org/licenses/by/4.0/.

(C) The Author(s) 2018 